\title{
Lung Cancer as a Second Primary Malignancy: Increasing Prevalence and Its Influence on Survival
}

\author{
Silvia Quadrelli ${ }^{1,2}$, Gustavo Lyons ${ }^{1}$, Henri Colt ${ }^{3}$, Domingo Chimondeguy ${ }^{1}$, and Carlos Silva ${ }^{1}$ \\ ${ }^{1}$ Thoracic Oncology Center, British Hospital, 1280 Buenos Aires, Argentina; ${ }^{2} 1425$ Buenos Aires, Argentina; ${ }^{3}$ Pulmonary \\ and Critical Care Unit, University of California Irvine Medical Center, Irvine, CA 92967
}

\begin{abstract}
Background. The purpose of this study was to review the experience with lung cancer as a second primary malignancy (LCSPM) and to determine whether a history of previous malignancy was an independent prognostic factor in resected non small cell lung cancer (NSCLC).

Methods. We included 414 patients undergoing pulmonary resection with a curative intention between January 1986 and January 2007. The criteria for classifying a lesion as a lung cancer as a second primary malignancy (LCSPM) were a different histological type or a tumor of identical histological type occurring more than 3 years after treatment of the primary tumor, or separated from the initial primary tumor by more than $2 \mathrm{~cm}$ of clinically normal epithelium.
\end{abstract}

Results. Thirty-four patients (8.2\%) had a history of a previous malignancy. LCSPM were more frequent during the last decade $(11.4 \%$ vs. $3.91 \%, P=.0009)$. Breast, uroepithelial, and lung cancer were the most prevalent initial primary tumors. In $64.7 \%$ of patients the time interval between the last malignancy and the current lung cancer was 2 years or shorter. Patients with NSCLC and a previous malignancy did not have a significantly different survival rate than patients with the current NSCLC as the first malignancy (5-year survival $65.3 \%$ vs. $58.6 \%$, logrank $P=.416$ ).

Conclusion. The effectiveness of the surgical resection and the subsequent survival of these patients appear to be well demonstrated, and operative mortality and complications are no higher than for other lung cancer patients.

(C) The Author(s) 2009. This article is published with open access at Springerlink.com

First Received: 8 August 2008;

Published Online: 4 February 2009

S. Quadrelli

e-mail: silvia.quadrelli@gmail.com
There is a paucity of studies pertaining to non-small cell lung cancer (NSCLC) as a secondary or tertiary primary tumor, including those with primary tumors from a nonpulmonary origin. Nor is there much published information about the influence of a history of a previous malignancy on survival in these patient groups. ${ }^{1-3}$ The purpose of this study, therefore, was to review our experience with lung cancer as a second primary malignancy (LCSPM) during the last 20 years and to determine whether a history of previous malignancy was an independent prognostic factor in resected NSCLC.

\section{METHODS}

All patients undergoing pulmonary resection with a curative intention for NSCLC in the British Hospital in Buenos Aires between January 1986 and January 2007 were eligible for inclusion in this retrospective study based on chart review. Patients with small cell lung cancer, rare histology, and those who had undergone neoadjuvant chemotherapy were excluded. The patients included in IIIB or IV Stage were patients with T4 and/or with solitary brain metastasis that were considered potentially resectable. The study was approved by the hospital's institutional review board. Survival status was obtained from telephone interviews and from the medical records.

No systematic screening for primary lung cancers was performed.

According to hospital protocol, preoperative staging was performed using TNM classification system of the International Union Against Cancer using chest computed tomography (CT) and abdominal CT or ultrasonography in all patients. Computed tomography or magnetic resonance imaging of the brain were done only in cases of clinical suspicion of brain metastases. In cases of uncertain clinical or radiologic findings, further examinations were performed to exclude extrapulmonary metastases. Mediastinal 
and hilar lymph node status was assessed as positive if the chest CT showed that the shorter axis of any node was larger than $1.0 \mathrm{~cm}$. A positron emission tomographic (PET) scan was not routinely performed. Mediastinoscopy has not been performed routinely in this series unless the CT scan demonstrated mediastinal lymph node enlargement. The clinicopathological records of each patient were examined for prognostic factors such as age, sex, right or left side cancer, histology, tumor location (upper or lower), tumor size, $\mathrm{cN}$ number, and distribution of metastatic nodes.

A history of previous malignancy was recorded only when the definite histological diagnosis of that primary tumor was available from the medical record. The criteria for classifying a lesion as second primary malignancy (LCSPM) followed Warren and Gates criteria. ${ }^{4}$ According to these criteria, a new cancer of different histological type, one of identical histological type occurring more than 3 years after treatment of the primary tumor, or one separated from the initial primary tumor by more than $2 \mathrm{~cm}$ of clinically normal epithelium were considered LCSPM. Local recurrences of lung metastasis and synchronous lung tumors were excluded from the analysis. ${ }^{4}$ Recurrences were considered to be local if they were found in the previous operative field (in the surgical incision), adjacent pulmonary parenchyma if a wedge or segmental resection was performed, bronchial stump if a lobectomy or pneumonectomy was performed, or hilar or mediastinal nodes on the same side as the primary tumor.

During surgery, bronchopulmonary, hilar, and mediastinal lymph nodes were systematically sampled. After surgery, a final pathologic stage was determined based on operative findings. Histologic typing was determined according to the World Health Organization classification. ${ }^{5}$ Lymph nodes were numbered and classified following Mountain's classification. ${ }^{6}$

Surgical resection was considered complete if all of the following criteria were present: free resection margins proved microscopically, systematic nodal dissection, no extracapsular nodal extension of the tumor, and the highest mediastinal node removed were negative. ${ }^{7}$

Postoperative follow-up included office visits, quarterly chest X-rays, and a yearly chest CT. Operative or in-hospital mortality was defined as death occurring within 30 days after the operation or during hospitalization, respectively. All patients with postoperative or in-hospital mortality were included in this study. Time interval was defined as the number of days between the day of surgery for the first tumor and the date of the diagnosis of the second or third lung cancer.

Statistical analysis was performed using SPSS 13.0 statistical software. The analysis of differences in categorical outcomes was determined using the chi-square test or Fisher exact test. Logistic regression models were used to ascertain the association between individual factors and survival. Probabilities of survival rates were estimated using the Kaplan-Meier method. Cox proportional hazards regression models were used to ascertain the association between individual factors and survival. Their joint effect was assessed in a multivariable Cox analysis. Statistical significance was assumed at $P<.05$. $^{8,9}$

\section{RESULTS}

We included 414 patients with NSCLC in this study. Of these, $34(8.2 \%)$ had a history of a previous malignancy. There were 39 tumors in 34 patients (one patient had three previous tumors, and three other patients had two previous tumors). If the 2 decades of this series are analyzed independently (1986-1996 vs. 1997-2006), LCSPM were more frequent during the last decade $(11.4 \%$ vs. $3.91 \%$, $P=.0009$ ). Breast, uroepithelial, and lung cancer were the most prevalent initial primary tumors (Table 1). In 23 patients $(71.9 \%)$, at least one of the previous malignancies was related to tobacco exposure, including primary tumors from the lung, uroepithelium, renal cell, gastric, and ENT (Table 2). The median time interval between the diagnosis of the first and second primary tumor was 32 months, (median, rank 1-296 months). The time interval was not different in patients with a previous malignancy related to tobacco exposure $(46.67 \pm 48.64$ vs. $67.73 \pm 85.09$ months, $P=.380)$. In 22 patients $(64.7 \%)$, the time interval between the last malignancy and the current lung cancer was 2 years or shorter (Table 2). Clinical characteristics of patients with a previous malignancy are shown in Table 2. Most patients had very limited disease $(79.4 \%$ of patients were Stage I) (Table 3).

Patients with a previous malignancy tended to be older (64.7 \pm 10.1 vs. $60.8 \pm 9.6$ years, $P=.036$ ), but sex distribution (male $61.7 \%$ vs. $75.5 \%, P=.110$ ) and the

TABLE 1 Location of the previous malignancy

\begin{tabular}{lll}
\hline Location of the first malignancy & $\mathrm{n}(n=39)$ & $\%$ \\
\hline Uroepithelium $^{\mathrm{a}}$ & 8 & 20.5 \\
Breast cancer $^{\mathrm{a}}$ & 8 & 20.5 \\
Lung cancer $^{\mathrm{a}}$ & 6 & 15.4 \\
Head and neck cancer $^{\mathrm{a}}$ & 4 & 10.3 \\
Colorectal cancer $^{\mathrm{a}}$ & 4 & 10.3 \\
Melanoma & 3 & 7.7 \\
Endometrium & 2 & 5.0 \\
Prostate cancer $_{\text {Gastric cancer }}^{\mathrm{a}}$ & 1 & 2.5 \\
Lymphoma/leukemia/myeloma $_{\text {Thyroid cancer }}$ & 1 & 2.5 \\
\end{tabular}

a Tobacco exposure related tumors 
TABLE 2 Clinical characteristics of patients with a previous malignancy $(n=34)$

\begin{tabular}{lc}
\hline Variables & $n(\%)$ \\
\hline Smokers & $9(28.1)$ \\
Never & $23(71.9)$ \\
Current/former & $12(35.3)$ \\
Tumor size $>3 \mathrm{~cm}$ & $11(32.4)$ \\
Lower lobe location & $6(17.6)$ \\
Central location & \\
Surgical procedures & $5(14.7)$ \\
Pneumonectomy & $29(85.3)$ \\
Lobectomy & \\
Side of lesion & $23(67.6)$ \\
Right & $11(32.4)$ \\
Left & \\
Histology & $23(67.6)$ \\
Adenocarcinoma & $2(5.9)$ \\
Adenosquamous carcinoma & $1(2.9)$ \\
Bronchoalveolar carcinoma & $6(7.6)$ \\
Squamous cell carcinoma & $2(5.9)$ \\
Large cell carcinoma & \\
Interval between tumors & $22(64.7)$ \\
$<2$ years & $12(35.3)$ \\
$>2$ years &
\end{tabular}

TABLE 3 Extension of the current disease in patients with a LCSPM

\begin{tabular}{lc}
\hline Extension variables & $n(\%)$ \\
\hline Clinical staging & \\
Stage IA & $21(61.8)$ \\
Stage IB & $6(17.6)$ \\
Stage IIB & $3(8.8)$ \\
Stage IIIA & $2(5.9)$ \\
Stage IIIB & $1(2.9)$ \\
Stage IV & $1(2.9)$ \\
Pathological staging & \\
Stage IA & $17(50)$ \\
Stage IB & $6(17.6)$ \\
Stage IIA & $2(5.9)$ \\
Stage IIB & $2(5.9)$ \\
Stage IIIA & $4(11.8)$ \\
Stage IIIB & $2(5.9)$ \\
Stage IV & $1(2.9)$ \\
\hline
\end{tabular}

proportion of smokers or ex-smokers $(64.7 \%$ vs. $73.6 \%$, $P=.356)$ were not significantly different. Patients with a previous malignancy had a more frequent incidence of solitary pulmonary nodule $(50 \%$ vs. $29.2 \%, P=.020)$ as the initial presentation of the current lung tumor and were symptomatic less frequently ( $25.5 \%$ vs. $43.9 \%, P=0.033)$.
No significant differences were noted among other clinical and radiological variables (Table 4).

The proportion of patients in whom resection was considered complete was not different $(91.1 \%$ vs. $85.2 \%$, $P=.490)$, nor was prevalence of metastatic mediastinal node involvement (pN2: $11.7 \%$ vs. $22.3 \%, P=.220$ ). However, the proportion of patients in Stage I (A or B) was larger in the group with a previous malignancy $(67.6 \%$ vs. $48.4 \%, P=.048)$. The proportion of patients with recurrence during follow-up was not different between the two groups $(29.4 \%$ vs. $35.5 \%, P=.597)$. Among those patients who had a recurrence, the frequency of distant metastasis (rather than local-regional recurrence) was not higher in the previous malignancy group $(20.5 \%$ vs. $24.4 \%$, $P=.765)$.

Operative morbidity was not different in patients with a previous malignancy (9.6 vs. $4.0 \%$, Fisher exact test $P=.094)$. Nonlethal morbidity included two cases of pneumonia, one air leak longer than 8 days, one wound infection, and one case of bleeding from duodenal ulcer. There was only one operative death in the previous malignancy group resulting in a similar operative mortality rate in both groups (5.8 vs. $4.3 \%, P=.709$ ).

Kaplan-Meier survival curves after the diagnosis of NSCLC in the two different groups are shown in Fig. 1. Patients with NSCLC and a previous malignancy did not have a significantly different survival rate than patients with the current NSCLC as the first malignancy (5-year survival $65.3 \%$ vs. $58.6 \%$, log-rank $P=.416$ ). If analysis is restricted to Stage I patients, survival is still the same (5-year survival $71.1 \%$ vs. $73.3 \%$, log-rank test $P=.803$ ) for the two groups (Fig. 2).

In the group of patients with a previous malignancy, neither the disease-free interval between the last tumor and the current lung cancer shorter than 2 years (odds ratio [OR] 2.252, confidence index [CI] 0.3246-15.6226, $P=.411$ ), nor a previous malignancy related to tobacco exposure (OR 0.9603, CI 0.1421-6.4882, $P=.966$ ) increased the risk of mortality at 5 years.

\section{DISCUSSION}

The prevalence of two or more malignancies in one patient has increased over the past decades. The proportion of second- or higher-order invasive cancers reported to the US International Cancer Institute's Surveillance, Epidemiology, and End Results (SEER) Program has increased from approximately $6 \%$ in 1973 to $15.1 \%$ in 1998 , while in Germany, an incidence of 1.2 per 100,000 inhabitants for lung cancer as a second primary malignancy after extrapulmonary primary tumors has been demonstrated. ${ }^{10}$ Our single-institution study adds to the body of evidence 
TABLE 4 Comparison of patients with and without a previous malignancy

\begin{tabular}{llll}
\hline Variable & $\begin{array}{l}\text { Patients with a } \\
\text { previous malignancy } \\
(n=34)(\%)\end{array}$ & $\begin{array}{l}\text { Patients without } \\
\text { a previous malignancy } \\
(n=380)\end{array}$ & $P$ \\
\hline Location right side & 67.6 & 58.1 & .370 \\
Central (CT scan) & 17.6 & 27.6 & .290 \\
Adenocarcinoma & 73.5 & 62.3 & .267 \\
Clinical N2 & 11.7 & 22.3 & .220 \\
Proportion of recurrence & 29.4 & 35.5 & .597 \\
Proportion of distant metastasis & 20.5 & 24.4 & .765 \\
Incidence of solitary pulmonary node & 50 & 29.2 & .020 \\
Resections was considered complete & 91.1 & 85.2 & .490 \\
Symptomatic & 25.5 & 43.9 & .033 \\
Proportion Stage I & 67.6 & 48.4 & .048 \\
Operative mortality & 5.8 & 4.3 & .709 \\
\hline
\end{tabular}

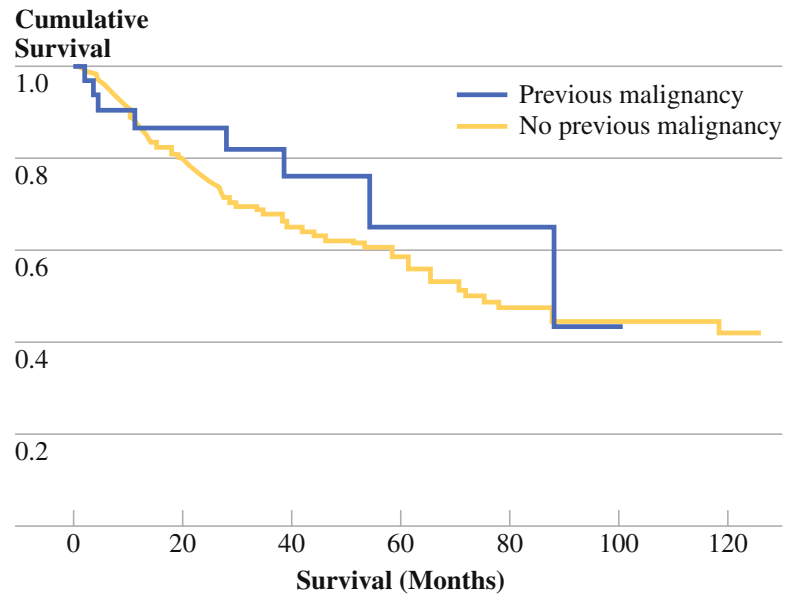

FIG. 1 Patients with NSCLC and a previous malignancy did not have a significantly different survival rate than patients with the current NSCLC as the first malignancy (5-year survival $65.3 \%$ vs. $58.6 \%, \log$-rank $P=.416$ )

demonstrating a greater prevalence of LCSPM in Argentina during the last decade $(11.4 \%$ vs. $3.91 \%, P=.0009)$.

Several hypotheses have been proposed to explain this increased frequency. These include the overall increase of life expectancy, which affects the prevalence of malignancies in general, and the increased use of radiation and/ or chemotherapy of first tumors, which might actually increase risk for secondary cancer. Van Leeuwen et al. for example noted an increased risk of lung cancer after mantle field irradiation for Hodgkin's disease, with the relative risk slowly increasing during the first 15 years after radiotherapy. ${ }^{11,12}$ An increased risk of lung cancer in the ipsilateral lung after breast cancer radiotherapy with a superadditive effect in smokers, relative risk (32.7) has also been reported. ${ }^{13-15}$

Smoking alone is also a potential risk factor for a secondary tumor. Johnson et al. ${ }^{16}$ noted that the risk of a new

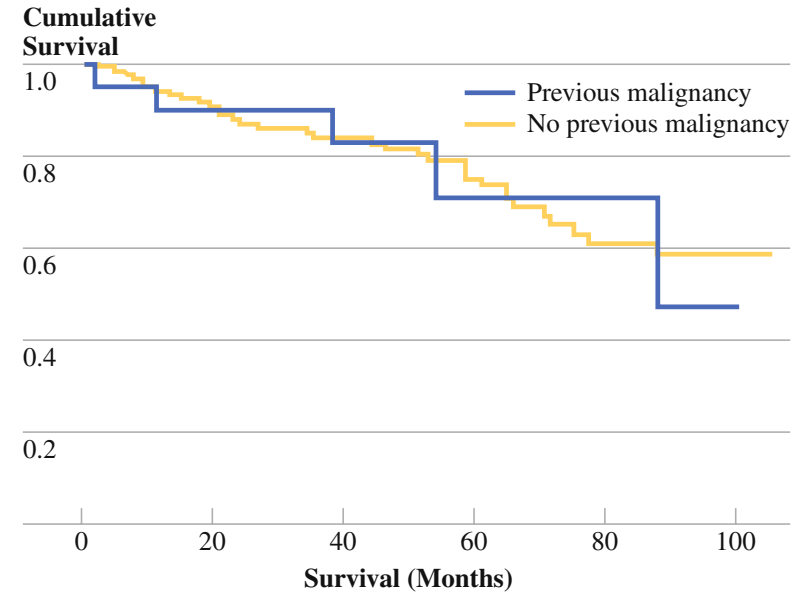

FIG. 2 Patients with NSCLC and a previous malignancy did not have a significantly different survival rate than patients with the current NSCLC as the first malignancy if is restricted only to sage I (5-year survival $71.1 \%$ vs. $73.3 \%$, log-rank test $P=.803$ )

primary tumor in smokers with a history of previous lung cancer is tenfold greater than for smokers without such a history, and finding asymptomatic potentially resectable second primaries is arguably a major reason for close follow-up in patients with successfully resected NSCLC. ${ }^{17}$

In regard to clinical follow-up, a prospective study of 346 patients with pathologically documented Stage I nonsmall cell lung cancer found that by using clinical followup two to three times per year with history and physical examination, blood studies, sputum cytology, and chest radiographs, 27 LCSPM were detected, $86 \%$ of which were asymptomatic. ${ }^{18}$ Of these, $77 \%$ were identified at the time of a bi- or tri-yearly scheduled examination.

This was also the case in our patients with LCSPM, who were more frequently found to have a solitary pulmonary nodule (50\% vs. $29.2 \%, P=.020)$ as the initial presentation of their current lung tumor, were symptomatic much 
less frequently $(25.5 \%$ vs. $43.9 \%, P=.033)$ and were more frequently diagnosed in Stage I (A or B). We believe this is explained by the fact that in our institution, cancer patients are subjected to thorough examination and followup in order to locate possible metastases or locoregional recurrences and that such close follow-up increases the possibility of finding a new lung cancer earlier.

Almost two-thirds of the patients with LCSPM in our study were prior or current smokers, but the proportion of smokers was not significantly different in patients with a first primary lung cancer $(64.7 \%$ vs. $73.6 \%, P=.356)$. Although $71.9 \%$ of the patients with LCSPM had a least one previous malignancy considered related to tobacco exposure, adenocarcinoma was the most prevalent histological type for the current lung cancer (67.6\%).This result is different from those of other studies that found squamous cell lung cancer to be the most prevalent histological type in LCSPM. ${ }^{3,19,20}$ It is noteworthy that other investigators have also found adenocarcinoma to be the most commonly discovered histology for second cancers. ${ }^{21,22}$

Of course, a LCSPM-proven adenocarcinoma opens the debate about the tumor's primary or secondary nature. There is evidence to support surgical resection in case of a solitary metastasis. According to data drawn from the International Registry, survival rates of $36 \%$ at 5 years following complete resection may be expected, ${ }^{23}$ and consequently, the unconfirmed suspicion of a metastatic lesion should not change surgical alternatives.

Results from epidemiological studies show a large proportion of patients with synchronous LCSPM, a fact that seems to prove that the occurrence of two primary malignancies in the same individual represents not only the consequence of adverse effects of previous tumor therapies (radiotherapy/chemotherapy), but it may also be of multifactorial origin. Potential determinants include not only smoking status but also lifestyle, (alcohol and diet, environmental causes) genetic susceptibility, immune function, and host hormonal status. ${ }^{24}$

This finding seems to have been reflected in our group of patients in whom, in spite of the high prevalence of a previous malignancy related to tobacco exposure, there were several types of primary cancers. One of the few differences between LCSPM and patients with a first cancer in our group was that patients with a previous malignancy were older. Although age is probably a simple surrogate for the duration of cigarette smoking, older patients might also have acquired greater genetic damage and be more susceptible to mutagenic effects.

Having the history of a previous malignancy in a resectable NSCLC does not increase the risk of incomplete resections and confers a 5-year survival rate that is similar to that of patients with a first lung primary tumor. Most authors have reported a similar ${ }^{25,26}$ or even better survival in resected LCSPM. Duchateau et al. reported that patients with more than two primary tumors were found to have a significantly better survival rate. The 5-year survival rate is significantly better for patients with more than two primary malignancies compared with patients without two primary malignancies and patients with one other tumor in their history $\left(P=.004\right.$ and .012 , respectively) ${ }^{3}$ Similarly, Liu et al. showed that the median survival of patients with a lung cancer as a second primary malignancy was better than the general lung cancer population. ${ }^{21}$ In a large epidemiological study, Hofman et al. reported survival rates of patients with LCSPM that were slightly better than the survival of patients with primary bronchial cancer and that patients with LCSPM, having undergone curative treatment, turned out to have the best prognosis overall, with a 5-year survival rate of $35 \% .^{10}$

Evidence about survival in resected LCSPM, however, is controversial. One explanation for the comparatively good survival figures in LCSPM, for example, is the greater proportion of patients in Stage I, but when only Stage I individuals were considered for the comparisons in our study, the 5-year survival showed no difference from those patients without a previous malignancy. It is noteworthy, therefore, that a large prospective study in Europe showed that the presence of a previous tumor represented an 1.5 increased risk of mortality at 5 years, particularly in patients with resected Stage I lung cancer (95\% CI 1.17$1.79) .^{20}$

It has been suggested that patients with two or more cancers have a predisposition to have tumors with a rather slow progression, ${ }^{3}$ but this evidence is inconclusive. It seems that the prognosis for patients suffering from LCSPM and an initial extrapulmonary tumor is mostly determined by the LCSPM and not by the initial extrapulmonary tumor. This hypothesis is mainly based on the fact that patients who died showed classic findings of metastatic spread of their bronchial carcinoma (lung, adrenal, liver, bone and brain). ${ }^{27}$

In summary, the results from our study add to the body of evidence suggesting that patients with a LCSPM and adequate pulmonary reserve should undergo appropriate pulmonary resections for their current lung cancers. The effectiveness of the surgical resection and the subsequent survival of these patients appears to be well demonstrated, and operative mortality and complications are no higher than for other lung cancer patients.

OPEN ACCESS This article is distributed under the terms of the Creative Commons Attribution Noncommercial License which permits any noncommercial use, distribution, and reproduction in any medium, provided the original author(s) and source are credited. 


\section{REFERENCE}

1. Martini N, Melamed MR. Multiple primary lung cancers. J Thorac Cardiovasc Surg. 1975;70:606-12.

2. Johnson BE. Second lung cancers in patients after treatment for an initial lung cancer. J Natl Cancer Inst. 1998;90:1335-45.

3. Duchateau CS, Stokkel MP. Second primary tumors involving non-small cell lung cancer: prevalence and its influence on survival. Chest. 2005; 127:1152-8.

4. Warren S, Gates O. Multiple primary malignant tumors. Am J Cancer. 1932;16:1358-1414.

5. Travis WD, Colby TV, Corrin B, Shimosato Y, Brambilla E. World Health Organization: Histological Typing of Lung and Pleural tumours. 3rd ed. Berlin: Springer, 1999.

6. Mountain CF, Dresler CM. Regional lymph node classification for lung cancer staging. Chest. 1997;111:1718-23.

7. Rami-Porta R, Wittekind C, Goldstraw P, International Association for the Study of Lung Cancer (IASLC) Staging Committee. Complete resection in lung cancer surgery: proposed definition. Lung Cancer. 2005;49:25-33.

8. Kaplan E, Meier P. Non-parametric estimation from incomplete observations. J Am Stat Assoc. 1958;53:457-81.

9. Cox D. Regression models and life-table (with discussion). $J R$ Statist Soc Series B. 1972;34:187-220.

10. Hofmann HS, Neef H, Schmidt P. Primary lung cancer and extrapulmonary malignancy. Eur $J$ Cardiothorac Surg. 2007;32:653-8.

11. van Leeuwen FE, Somers R, Taal BG, van Heerde P, Coster B, Dozeman $\mathrm{T}$, et al. Increased risk of lung cancer, non-Hodgkin's lymphoma, and leukemia following Hodgkin's disease. J Clin Oncol. 1989;7:1046-58.

12. van Leeuwen FE, Klokman WJ, Hagenbeek A, Noyon R, van den Belt-Dusebout AW, van Kerkhoff EH, et al. Second cancer risk following Hodgkin's disease: a 20-year follow-up study. J Clin Oncol. 1994;12:312-25.

13. Neugut AI, Robinson E, Chul Lee W, Murray T, Karwoski K, Kutcher J. Lung cancer after radiotherapy for breast cancer. Cancer. 1993;71:3054-7.

14. Inskip PD, Stovall M, Flannery JT. Lung cancer risk and radiation dose among women treated for breast cancer. $J$ Natl Cancer Inst. 1994;86:983-8.
15. Neugut AI, Murray T, Santos J, Amols H, Hayes MK, Flannery JT, et al. Increased risk of lung cancer after breast cancer radiation therapy in cigarette smokers. Cancer. 1994;73:1615-20.

16. Johnson BE, Cortazar P, Chute JP. Second lung cancers in patients successfully treated for lung cancer. Semin Oncol. 1997;24:492-9.

17. Shields TW. Postoperative lung cancer surveillance: who, what, when, and why? Chest. 1997;111:11-12.

18. Pairolero PC, Williams DE, Bergstralh EJ, Piehler JM, Bernatz PE, Payne WS. Postsurgical stage I bronchogenic carcinoma: morbid implications of recurrent disease. Ann Thorac Surg. 1984;38:331-8.

19. Massard G, Ducrocq X, Beaufigeau M, Elia S, Kessler R, Hervé $\mathrm{J}$, et al. Lung cancer following previous extrapulmonary malignancy. Eur J Cardiothorac Surg. 2000;18:524-8.

20. López-Encuentra A, Gómez de la Cámara A, Rami-Porta R, Duque-Medina JL, de Nicolás JL, Sayas J, Bronchogenic Carcinoma Cooperative Group of the Spanish Society of Pneumology and Thoracic Surgery (GCCB-S). Previous tumour as a prognostic factor in stage I non-small cell lung cancer. Thorax. 2007;62:386-90.

21. Liu YY, Chen YM, Yen SH, Tsai CM, Perng RP. Multiple primary malignancies involving lung cancer-clinical characteristics and prognosis. Lung Cancer. 2002;35:189-94.

22. Asaph JW, Keppel JF, Handy JR, Douville C, Tsen AC, Ott GY. Surgery for second lung cancers. Chest. 2000;118:1621-5.

23. The International Registry of Lung Metastases. Long-term results of lung metastasectomy: prognostic analyses based on 5206 cases. J Thorac Cardiovasc Surg. 1997;113:37-49.

24. Travis LB. Therapy-associated solid tumors. Acta Oncol. 2002;41:323-33.

25. Furák J, Troján I, Szöke T, Wolfárd A, Nagy E, Németh I, et al. Lung cancer as a second primary malignant tumor: prognostic values after surgical resection. Interact Cardiovasc Thorac Surg. 2008;7:50-3.

26. Koppe MJ, Zoetmulder FA, van Zandwijk N, Hart AA, Baas P, Rutgers EJ. The prognostic significance malignancy in operable non-small cell lung cancer. Lung Cancer. 2001;32:47-53.

27. Samuel A, Adebonojo SA, Moritz DM, Danby CA. The results of modern surgical therapy for multiple primary lung cancers. Chest. 1997;112:693-701. 